\title{
Diffusion tensor imaging as a biomarker for assessing neuronal stem cell treatments affecting areas distal to the site of spinal cord injury
}

\author{
Michael B. Jirjis, PhD, ${ }^{1,3}$ Chris Valdez, PhD, ${ }^{4}$ Aditya Vedantam, MD, ${ }^{2}$ Brian D. Schmit, $\mathrm{PhD},{ }^{1}$ and \\ Shekar N. Kurpad, MD, PhD²
}

1Department of Biomedical Engineering, Marquette University; ${ }^{2}$ Department of Neurosurgery, Medical College of Wisconsin, Milwaukee, Wisconsin; and ${ }^{3}$ Air Force Research Laboratory, 711th Human Performance Wing, Human Effectiveness Directorate, Bioeffects Division, Radio Frequency Bioeffects Branch, and ${ }^{4}$ National Research Council Research Associateship Program, Joint Base San Antonio, Fort Sam Houston, San Antonio, Texas

OBJECTIVE The aims of this study were to determine if the morphological and functional changes induced by neural stem cell (NSC) grafts after transplantation into the rodent spinal cord can be detected using MR diffusion tensor imaging (DTI) and, furthermore, if the DTI-derived mean diffusivity (MD) metric could be a biomarker for cell transplantation in spinal cord injury (SCI).

METHODS A spinal contusion was produced at the T-8 vertebral level in 40 Sprague Dawley rats that were separated into 4 groups, including a sham group (injury without NSC injection), NSC control group (injury with saline injection), coinjection control group (injury with Prograf), and the experimental group (injury with NSC and Prograf injection). The NSC injection was completed 1 week after injury into the site of injury and the rats in the experimental group were compared to the rats from the sham, NSC control, and co-injection groups. The DTI index, MD, was assessed in vivo at 2, 5, and 10 weeks and ex vivo at 10 weeks postinjury on a 9.4-T Bruker scanner using a spin-echo imaging sequence. DTI data of the cervical spinal cord from the sham surgery, injury with saline injection, injury with injection of Prograf only, and injury with C17.2 NSC and Prograf injection were examined to evaluate if cellular proliferation induced by intrathoracic C17.2 engraftment was detectable in a noninvasive manner.

RESULTS At 5 weeks after injury, the average fractional anisotropy, longitudinal diffusion (LD) and radial diffusion (RD) coefficients, and MD of water (average of the RD and LD eigenvalues in the stem cell line-treated group) increased to an average of $1.44 \times 10^{-3} \mathrm{sec} / \mathrm{mm}^{2}$ in the cervical segments, while the control groups averaged $0.98 \times 10^{-3} \mathrm{~s} / \mathrm{mm}^{2}$. Post hoc Tukey's honest significant difference tests demonstrated that the transplanted stem cells had significantly higher MD values than the other groups ( $p=0.032$ at 5 weeks). In vivo and ex vivo findings at 10 weeks displayed similar results. This statistical difference between the stem cell line and the other groups was maintained at the 10 -week postinjury in vivo and ex vivo time points.

CONCLUSIONS These results indicate that the DTI-derived MD metric collected from noninvasive imaging techniques may provide useful biomarker indices for transplantation interventions that produce changes in the spinal cord structure and function. Though promising, the results demonstrated here suggest additional work is needed before implementation in a clinical setting.

https://thejns.org/doi/abs/10.3171/2016.5.SPINE151319

KEY WORDS diffusion tensor imaging; C17.2 neuronal stem cells; spinal cord injury; magnetic resonance imaging

ABBREVIATIONS BBB = Basso, Beattie, and Bresnahan; CGRP = calcitonin gene-related peptide; DAPI = 4,6'-diamino-2-phenylindole-dihydrochloride; DTI = diffusion tensor imaging; FA = fractional anisotropy; FOV = field of view; GAP43 = growth-associated peptide 43 ; LD = longitudinal diffusion; MD = mean diffusivity; NEX = number of excitations; NSC = neural stem cell; OD = optical density; PBS = phosphate-buffered saline; RD = radial diffusion; $\mathrm{ROI}=$ region of interest; $\mathrm{SCl}=\mathrm{spinal}$ cord injury. SUBMITTED November 5, 2015. ACCEPTED May 27, 2016.

INCLUDE WHEN CITING Published online September 30, 2016; DOI: 10.3171/2016.5.SPINE151319. 
$\mathrm{N}$ ONINVASIVE MRI has the potential to monitor cellular transplant intervention treatments in spinal cord tissue after spinal cord injury (SCI). The MR diffusion tensor imaging (DTI) technique is an appealing tool for monitoring the effects of cellular transplants in the spinal cord because, by measuring the changes in water diffusion associated with injury, DTI can provide data on microstructural changes in the spinal cord in both the acute and chronic stages of SCI. $8,9,16,20,33,37,39$ Previous reports have demonstrated that the water diffusion measurements recorded by DTI are associated with white matter regeneration following the injection of neural progenitor cells in stroke, as well as after the injection of fibroblast and autoimmune T-cell transplantation. ${ }^{12,27,32}$ The changes in the axonal structure and corresponding variations in water diffusion from these implants suggest that neuronal stem cells (NSCs) produce membrane structures that alter tissue integrity and will impact the diffusion of water measured by DTI. ${ }^{10}$

In this study, we sought to determine if DTI was sensitive to spinal cord tissue changes that accompany stem cell implants. We hypothesized that DTI would detect structural changes in the cervical region of the spinal cord that occur after the transplantation of C17.2 NSCs at a lower thoracic (T-8 level) contusion injury site. Our rationale for choosing the cervical imaging site was based on previous studies that have documented structural changes in the cervical spinal cord along with functionally altered pain perception in the forepaws following SCI. ${ }^{19,22}$ To address our goal, we conducted a longitudinal imaging study in which specimens were monitored for up to 10 weeks in vivo with DTI and then imaged ex vivo after euthanization at 10 weeks. The rats were tested for motor and sensory function, and end-of-life histology was examined using previously established methods. ${ }^{22}$ Mean diffusivity (MD), among other metrics, was compared between groups that received sham surgery, injury with saline injection, injury with injection of an immunosuppressant only, and injury with C17.2 NSC injection. Our results demonstrate that morphological changes in the cervical spinal cord induced by intrathoracic C17.2 NSC transplantation can be detected using DTI.

\section{Methods}

This study consisted of a spinal contusion injury, injection of stem cells or control solution, and survival for 10 weeks in a rat model. ${ }^{35}$ Sensory and motor tests were conducted over the course of the study, and DTI was performed both in vivo and ex vivo. Structural changes in the cervical spinal cord after intrathoracic C17.2 NSC transplantation were documented using immunohistochemical staining for calcitonin gene-related peptide (CGRP) and growth-associated peptide 43 (GAP43). ${ }^{11,21}$ The study protocol was approved under the guidelines of the Institutional Animal Care and Use Committees at Marquette University, the Medical College of Wisconsin, and the Zablocki Veterans Affairs Medical Center.

\section{Spinal Cord Injury}

For this study, a spinal contusion injury was produced in 40 adult female Sprague Dawley rats (weight range 200-250 g) that were approximately 50 days old. Rats were anesthetized prior to surgery using a $2 \%-3 \%$ isoflurane and oxygen mixture. The animals were placed prone, and an incision was made over the midthoracic region. Following incision, laminectomies were performed over the T7-9 vertebral levels, and a moderate contusion injury was produced in all groups but the sham group using the MASCIS impactor (W. M. Keck Center for Collaborative Neuroscience) with a $10-\mathrm{g}$ rod that was dropped from a height of $25 \mathrm{~mm}$ directly onto the dura. The sham group $(n=10)$ only received laminectomies without SCI. The postoperative regimen consisted of 1 dose of enrofloxacin (10 mg/kg administered subcutaneously; Bayer Healthcare LLC), buprenorphine hydrochloride $(0.1-0.5 \mathrm{mg} / \mathrm{kg}$ administered subcutaneously; Reckitt Benckiser Health Care Ltd.) as needed, $6 \mathrm{ml}$ of lactated Ringer's solution administered subcutaneously, and manual bladder expression twice per day.

\section{Cell Transplantation and Stereotactic Injection}

Spinal cord injections were performed 1 week after injury. Rats were reanesthetized using the same procedure that was used when producing the spinal injury, and the contusion site was reexposed for stereotactic injection. Rats were randomized into 4 groups to receive no transplant injection ( $n=10$; the sham group), sterile phosphatebuffered saline (PBS) ( $n=10$; NSC control group), sterile PBS with $50 \mathrm{mg} / \mathrm{kg}$ of the immunosuppressant drug tacrolimus (Prograf, Mylan Pharmaceuticals Inc.) $(n=10$; co-injection control group), or C17.2 NSCs with Prograf ( $n=10$; experimental group). ${ }^{38}$ Surgeons were unaware of which injection material was being used, and Prograf was used to suppress the immune response in order to prevent graft rejection of the C17.2 NSC line in this xenotransplant model. ${ }^{3}$

\section{NSC Line}

The C17.2 NSC line was used in the transplant group. The C17.2 NSC line was provided by the laboratory of Evan Y. Snyder, MD, PhD (Harvard University). ${ }^{31,36}$ Prior to surgery, $1 \times 10^{6}$ cells were counted using the trypan blue exclusion method and plated. Upon 80\%-100\% confluency, the cells were harvested with trypsin and washed with Dulbecco's Modified Eagle's Medium (Life Technologies) containing fetal bovine serum and a high glucose level. The cells were then washed with sterile PBS $(\mathrm{pH}$ 7.4), counted using trypan blue, centrifuged, and resuspended at $1 \times 10^{5}$ cells per $20 \mu$ l of sterile PBS. The cell suspension was placed on ice and gently titrated before each injection. The transplant injections $\left(5 \mu \mathrm{l} ; 2.5 \times 10^{4}\right.$ cells per injection) were delivered in a Kopf stereotactic frame into the spinal cord under microscopic visualization at 4 injection sites: $1 \mathrm{~mm}$ rostral and $1 \mathrm{~mm}$ caudal to the injury site, as well as on either side of the midline. The surgical site was closed in layers, and the animals received postoperative care as described above.

\section{Behavioral Testing}

Animals were tested on a weekly basis for locomotor 
function, thermal stimulation response, and mechanical stimulation response. Basso, Beattie, and Bresnahan (BBB) scoring was used to evaluate locomotor function by placing the rat on a flat surface that was $1 \mathrm{~m}$ in diameter and evaluating the hind limb function of the rat for 3 minutes. ${ }^{2}$ The rat was then scored between 0 (flaccid paralysis) and 21 (normal gait). The thermal response was measured by placing the rat on a hot $\left(55^{\circ} \mathrm{C}\right)$ plate and measuring the latency of the forelimb and hindlimb paw to withdraw from the heat. Animals that did not respond after 60 seconds were removed from the hot plate. Mechanical stimulation was recorded by measuring the number of withdrawal responses to the forelimb and hindlimb paw using Von Frey monofilament testing. Ten applications were randomly applied to the 4 paws using $3.92-\mathrm{mN}$, 9.8- $\mathrm{mN}$, and 254.9-mN filaments, which were delivered at 3 -second intervals.

\section{In Vivo DTI}

In vivo imaging was performed at 2,5, and 10 weeks following SCI. Magnetic resonance images were collected using the 9.4-T Bruker BioSpec 94/30 USR Spectroscopy Imaging System (Bruker BioSpin). The transmission and reception of radiofrequency signals was done using a quadrature coil (Doty Scientific). Animals were anesthetized with an isoflurane (1\%-1.5\%) and oxygen mixture and placed supine inside the scanner. A bite bar and head block were used to hold the head in place, and the body was nested inside a half-recessed plastic tube to keep movement contained. Core body temperature was maintained with a circulating warm water pad $\left(37^{\circ} \mathrm{C}\right)$, and $\mathrm{MR}$ images were respiration gated to avoid breathing artifacts that would induce motion. Respiration was monitored using the SA Instruments model 1025 MR-compatible monitoring and gating system (SA Instruments Inc.), and acquisition took place during the expiration phase.

Twelve noncollinear diffusion-weighted directions were collected using a spin-echo sequence with the following parameters: TE $=25.11 \mathrm{msec}$; TR $>600 \mathrm{msec}$ (reliant on the respiratory rate). Images were acquired with the following parameters: $\mathrm{b}=500 \mathrm{sec} / \mathrm{mm}^{2}$; number of excitations $(\mathrm{NEX})=2$; field of view $(\mathrm{FOV})=40 \mathrm{~mm}^{2}$; acquisition matrix $=128 \times 128$. Two diffusion sensitizing images with a b value of $0 \mathrm{sec} / \mathrm{mm}^{2}$ were acquired. Three images with a 2-mm-thick slices and a 1-mm interslice gap were acquired over the C6-T1 segments. This region of the spinal cord was selected because changes in histology, along with behavioral changes in the forepaws, have been associated with these spinal segments after cell transplant.22

\section{Ex Vivo DTI}

Following the 10 -week in vivo scan, the animals were killed with sodium pentobarbital (100 $\mathrm{mg} / \mathrm{kg}$ body weight) and perfused with $300 \mathrm{ml}$ of saline buffer followed by 600 $\mathrm{ml}$ of $10 \%$ formalin. The spinal cords were dissected and postfixed in $10 \%$ formalin. Ex vivo DT images of the extracted cords were obtained on the same scanner that was used for in vivo imaging. On the day of scanning, the spinal cords were embedded in agarose gelatin according to the protocol of Ellingson et al. ${ }^{6}$ Six diffusion-weighted images were collected using a spin-echo imaging sequence with the following parameters: $\mathrm{TE}=27.3 \mathrm{msec}$; $\mathrm{TR}=$ $6250 \mathrm{msec}$. The images were collected with the following parameters: $\mathrm{FOV}=70 \mathrm{~mm}^{2} ; \mathrm{NEX}=2$; matrix size $=$ $512 \times 512$. The diffusion images were acquired with a $b$ value of $500 \mathrm{sec} / \mathrm{mm}^{2}$, in addition to $2 \mathrm{~T} 2$-weighted images $\left(b=0 \mathrm{sec} / \mathrm{mm}^{2}\right)$. A total of 25 slices were obtained with 2-mm-thick slices and a 1-mm interslice gap over the entire spinal cord.

\section{Diffusion Tensor Data Analysis}

Calculation of the diffusion tensor data was conducted using AFNI (http://afni.nimh.nih.gov/afni). The RAW image files were imported into AFNI, and the diffusionweighted images were coregistered to the T2-weighted images using the iteratively weighted least squares fit method. Following registration, the tensor estimate was found using AFNI, and the resulting eigenvalues were used to calculate fractional anisotropy (FA), mean diffusivity (MD), the longitudinal diffusion (LD) coefficient (the primary eigenvalue), and the radial diffusion (RD) coefficient (the average of the second and third eigenvalues). ${ }^{1}$ FA is typically expressed as a range from 0 to 1 , and it describes how isotropic or anisotropic the diffusion of the water molecules is in that region. MD defines the overall diffusion from each of the 3 directions in the tensor, while $\mathrm{LD}$ and RD describe the diffusion in specific directions. Diffusion along the spinal cord (i.e., LD) can be associated with changes in axon diameter or damage to axons..$^{32}$ Diffusion in the radial direction has been associated with changes in myelin. ${ }^{37}$

Regions of interest (ROIs) were manually selected from each axial slice for both the in vivo and ex vivo images. Using MATLAB (MathWorks), the ROIs of the entire cord encompassing both the white and gray matter regions were traced for each axial slice, and regions within 2 voxels around the edge of the cord were excluded to avoid partial contamination from the surrounding cerebrospinal fluid.

\section{Immunohistochemical Analysis}

Following ex vivo imaging, 2 spinal cords per group were randomly selected for immunohistochemical analysis. The C6-T1 region of the spinal cord was identified and resected for fluorescent staining. The C6-T1 region was selected because altered pain perception in the forepaws and the cytoarchitectural changes in these regions are observed in specimens receiving spinal implantation of C17.2 cells..$^{18,28,36}$ Immunohistochemical analysis was performed using a previously described protocol. ${ }^{22}$ Tissue samples were postfixed in $4 \%$ paraformaldehyde/PBS at $4^{\circ} \mathrm{C}$ for an hour, frozen in OCT compound (Sakura Finetek USA, Inc.), and stored at $-80^{\circ} \mathrm{C}$ until sectioning. The tissues were sectioned into $20-\mu \mathrm{m}$-thick slices using a Leica CM3050 S cryostat (Leica Microsystems Inc.) and placed on Vectabond-coated slides.

The tissue sections were allowed to thaw for 1 hour before incubation in the primary antibodies. The sections were double labeled with a 1:1000 polyclonal CGRP 
(Chemicon International Inc.) and 1:1000 monoclonal GAP43 (Chemicon), followed by washing and then incubation with a 1:500 diluted goat anti-mouse conjugated fluorescein isothiocyanate (Jackson ImmunoResearch Inc.) and 1:500 diluted Alexa Fluor 594 goat anti-rabbit antibody (Invitrogen Corp.). All sections were counterstained with a 1:500 dilution of the nonspecific nuclear stain with 4,6'-diamino-2-phenylindole-dihydrochloride (DAPI) (Sigma-Aldrich Co.) and then imaged using a Nikon Eclipse E600 microscope equipped with a Nikon Digital Sight D5-U3 color camera at 40x magnification. Cell counting was completed using ImageJ (National Institutes of Health).

Dorsal horn densitometry was measured as the optical density (OD) of the dorsal horn region subtracted from the OD of the background. The images of the immunolabeled sections were converted to gray scale (0-255 arbitrary units), which represent the black to white intensity values, and ImageJ software was used to determine the resulting OD values for each rat around Laminae II-IV for slices in the C6-T1 vertebral segments.

\section{Statistical Analysis}

The statistical analysis was performed using the Statistical Package for Social Sciences (SPSS version 13.0; SPSS Inc.). Statistical significance was determined using repeated-measures 1-way ANOVA (the transplant group was the fixed factor, and the specimen was the random factor), followed by Fisher's post hoc test for the hot plate and Von Frey sensory data as well as the dorsal horn cell count measurements. BBB scoring used 1-way ANOVA followed by nonparametric Kruskal-Wallis and Friedman's rank tests with a level of significance of $\alpha=0.05$. Diffusion metrics were averaged at each slice for each specimen and then averaged across specimens. Repeated measures 2-way ANOVA (the transplant group and slice location [C6-T1] were fixed factors, and the specimen was the random factor) was also completed.

\section{Results}

\section{DTI of the Cervicothoracic Region}

Diffusivity was quantified by selecting ROIs that encompassed transverse slices through the spinal cord (Fig. 1). Rats that received the C17.2 NSC transplantation displayed significantly different diffusion values compared with the control groups $(\mathrm{p}<0.05)$ at multiple time points in terms of MD, LD, and RD (Fig. 2). In terms of FA, there was no significant difference between C17.2 NSC-treated rats and the other 3 groups (sham, NSC control group, and co-injection control group) at any time point ( $\mathrm{p}>0.05)$. MD at Weeks 5 and 10 and on the ex vivo scans showed that there was a significant increase in the diffusion of water when compared with the control groups $(\mathrm{p}=0.032$, 0.041 , and 0.019 , respectively). LD was also significantly greater at Weeks 5 and 10 and the ex vivo time points ( $\mathrm{p}$ $=0.023,0.026$, and 0.013 , respectively). At 5 weeks after injury, the average fractional anisotropy, longitudinal diffusion (LD) and radial diffusion (RD) coefficients, and $\mathrm{MD}$ of water (average of the RD and LD eigenvalues in the stem cell line-treated group) increased to an average

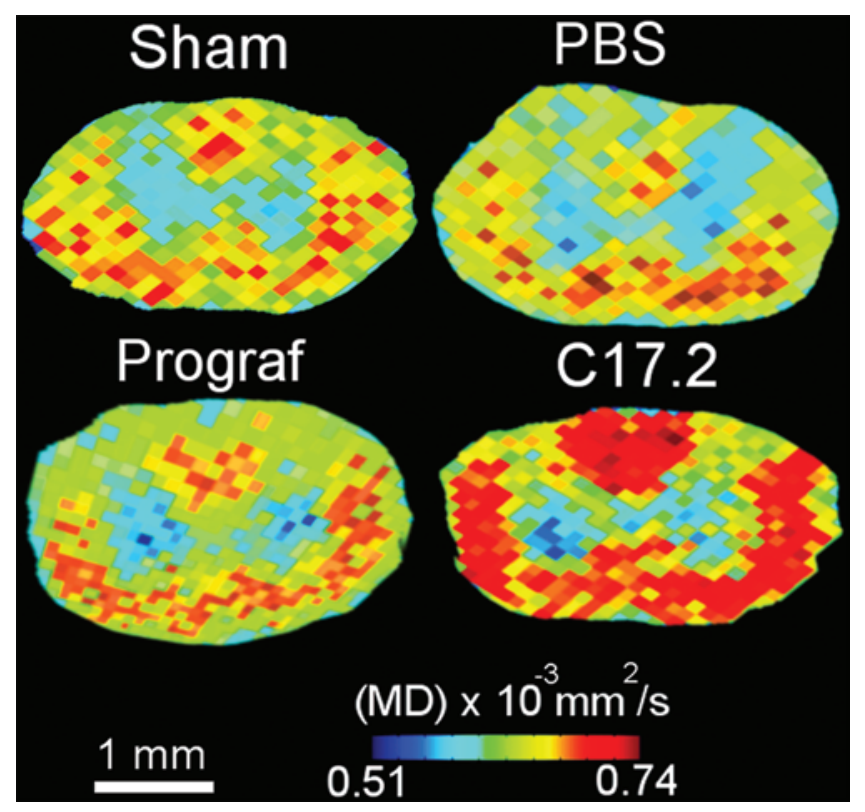

FIG. 1. Representative MR images of MD from the rat spinal cord from the sham, PBS, Prograf, and C17.2 groups. Images were taken at the C-7 vertebral segment in 4 rats (1 from each group) and were obtained ex vivo at 10 weeks after injury. Figure is available in color online only.

of $1.44 \times 10^{-3} \mathrm{sec} / \mathrm{mm}^{2}$ in the cervical segments, while the control groups averaged $0.98 \times 10^{-3} \mathrm{~s} / \mathrm{mm}^{2}$. The diffusion of water through the cord became significantly greater for the rats that received the C17.2 NSC line than the control rats at Week 10 and remained significant on the ex vivo scans $(\mathrm{p}=0.002)$.

\section{Immunohistochemical Characterization}

The C17.2 NSC group showed axonal sprouting in the dorsal horn (Laminae I-III) in the cervicothoracic (C7T1) region (Fig. 3). On the same image, this region also showed an increase in CGRP and GAP43 immunoreactivity. The C17.2 NSC group showed greater colocalization of CGRP with GAP43 in Laminae I-III compared with the other groups. The densitometry results from the immunolabeling of CGRP and GAP43 and the nuclear DAPI stain resulted in a significantly greater density $(\mathrm{p}=$ 0.0009) in Laminae II-IV when compared with the other groups (Fig. 4).

\section{Behavioral and Sensory Tests}

The hindlimb motor function (i.e., BBB score) of the rats that received PBS, Prograf only, or C17.2 NSC transplantation was significantly reduced compared with the sham group for Weeks 2 through 10 postsurgery $(\mathrm{p}=$ 0.0002-0.0004). As shown in Fig. 5, the rats that received an injection after injury were not significantly different than each other at all time points, in accordance with our previously published data $(\mathrm{p}>0.05),{ }^{22}$ and the sham group demonstrated no loss of motor function for the recorded weeks.

Rats that received C17.2 NSCs demonstrated a decrease in the withdrawal latency following thermal stimulation to the forelimbs. Following injection, the PBS and Prograf 


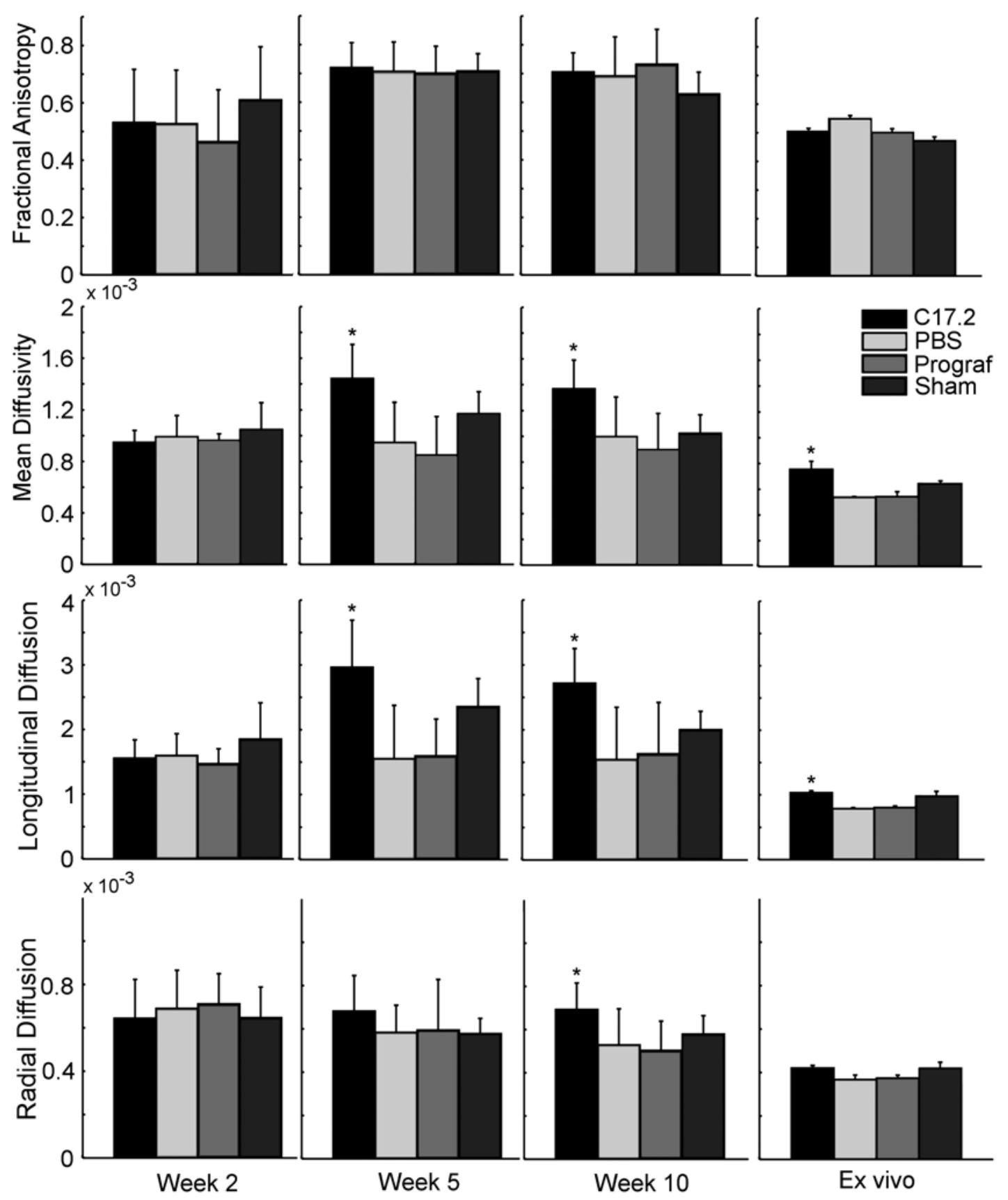

FIG. 2. In vivo DTI values for Weeks 2, 5, and 10 as well as for ex vivo imaging. The values come from ROls over the entire cord. The measurements were an average of 3 slices over the cervical segments at C6-T1. FA, MD, and the LD and RD coefficients are shown for each time point. Values are presented as the mean and standard error. The C17.2 NSC transplant group had a significantly higher MD at Weeks 5 and 10 and on the ex vivo scans compared with controls $(p<0.05)$. Both LD and RD showed a significantly higher rate of diffusion at Week 10 ( $\left.{ }^{*} p<0.05\right)$; however, LD also showed a significantly higher rate of diffusion at Week 5 and on the ex vivo scans.

groups had significantly longer withdrawal times than the sham group at multiple time points $(\mathrm{p}<0.05)$. As shown in Fig. 6, withdrawal latencies in the PBS and Prograf groups were not significantly different from each other $(p>0.05)$, but they were significantly different from the rats that received the C17.2 NSC transplantation at Weeks 3 through $6(\mathrm{p}=0.045,0.001,0.039$, and 0.034 , respectively $)$, as well as at Weeks 9 and $10(\mathrm{p}=0.032$ and 0.029 , respectively). Withdrawal latency decreased postinjection from 8 seconds to 4 seconds in the rats that received the stem cells over the 8 weeks of sensory recording. There were no significant differences in the response to mechanical stimulation in the 4 groups of rats, and thus those data are not shown. 


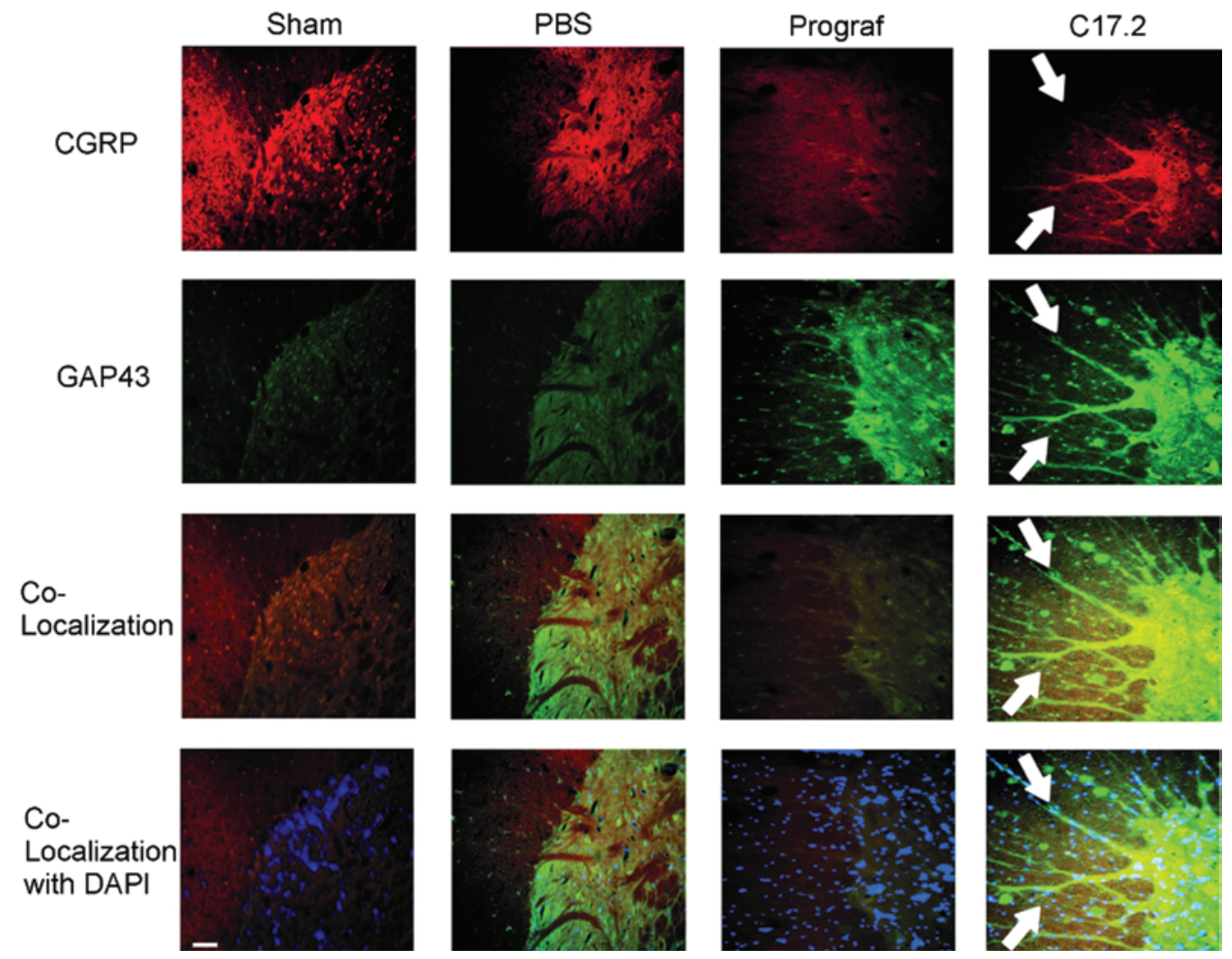

FIG. 3. Immunohistochemical labeling in the dorsal horns of the C7-T1 vertebral segments. Immunofluorescence is shown for CGRP (red), GAP43 (green), colocalization of CGRP and GAP43 (yellow), and colocalization of CGRP and GAP43 with nonspecific labeling of the cell nuclei with DAPI (blue). Axon sprouting (arrows) was demonstrated in the C17.2 NSC transplant group that was not seen in the sham, PBS, and Prograf groups. Magnification was set at $40 x$. Scale bar is $100 \mu \mathrm{m}$. Figure is available in color online only.

\section{Discussion}

The results from this study suggest that DTI is a viable tool for noninvasively monitoring the effects of cellular transplants in the spinal cord. To the best of our knowledge, this is the first study to use DTI as a biomarker for NSC transplantation. SCI rats were treated with stem cells and then evaluated over a 10 -week period. The cervical spinal cord in each rat was imaged in vivo at Weeks 2, 5, and 10 and ex vivo at Week 10 and then compared with functional measures of sensory and motor function in order to understand the temporal effects of NSC engraftment on the anatomical structure as well as terminal immunohistological studies. The results demonstrated changes in $\mathrm{MD}, \mathrm{LD}$, and RD that appeared to be in agreement with thermal allodynia in the forepaws and tissue structural changes in the cervical spinal cord.

Stem cell transplantation after SCI manipulates the environment in a way that changes the rate of the diffusion of water through tissue. Following moderate injury, the motor functions of the injury groups were significantly reduced for the remaining 10 weeks. However, as shown in Fig. 3 and supported by previous research on C17.2 transplants, engrafted C17.2 stem cells differentiate into astrocytes, thereby eliciting forelimb allodynia. ${ }^{22,28}$ This increase in thermal sensitivity was significant at 3-6, 9, and 10 weeks following injury. Our results in this study replicate previ- ously published results, corroborating the development of forelimb allodynia after intrathoracic NSC transplants reported by Macias et al. and Hofstetter et al. ${ }^{12,22}$

The in vivo diffusion measurements in the cervical cord were first significantly different from the control groups at 5 weeks after injury and continued to be significant for the rest of the experiment. The diffusion measurements in both the longitudinal and transverse directions of the cord, as well as MD, showed significant differences at Weeks 5 and 10 as well as ex vivo, suggesting that DTI may detect structural changes. As shown in the supported immunohistochemical images in Fig. 3, there is a change in the biological structure of the environment following the implantation of stem cells. This biological change included astrocytic growth and changes in the density and distribution of CGRP (a membrane receptor marker that modulates highly integrated pain signaling in the spinal cord) and GAP43 (a cytoplasmic marker expressed on regenerating axons following SCI) ${ }^{11,21}$ Intrathoracic C17.2 NSC grafts have been histologically shown to create structural changes in the cervical segments as early as 10 days after injection. ${ }^{22}$ There was no significant difference in the in vivo diffusion measurements at 2 weeks after injury, suggesting that DTI is not more sensitive to structural changes than early functional testing.

Histological analysis of the spinal cord was consistent 


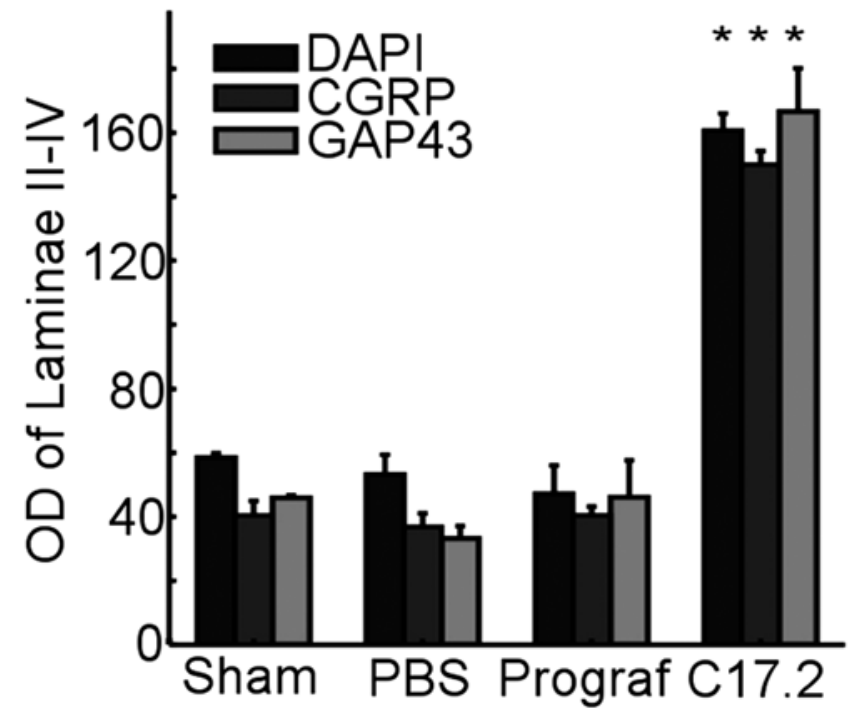

FIG. 4. Densitometric values of the immunolabeled CGRP and GAP43 stains based on OD. There was a statistically significant increase $(p<$ 0.05 ) in the density of CGRP and GAP43, as well as nuclei staining with DAPI $(p<0.05)$, when compared with the control groups.

with the tissue responses reported for the $\mathrm{C} 17.2$ transplants that produced allodynia. ${ }^{22,28,36}$ As shown in Figs. 3 and 4 , the C17.2 NSCs were characterized by an increase in CGRP and GAP43 sprouting from the dorsal horn, which is consistent with previous work. ${ }^{20}$ Sprouting has also been shown in distal segments following SCI and cell transplantation. ${ }^{11,22,29} \mathrm{MD}$ increases with respect to densitometry measurements, which was counterintuitive at first. However, after secondary degeneration, the liquid formation of necrotic tissue has been shown to increase MD, and it is also possible that the degenerated debris created by an SCI was removed more efficiently with the increase in the differentiation of NSCs into cells such as macrophages. ${ }^{30}$

While we suspect that histological changes underlie the changes in DTI metrics, there may be other factors involved. It has been suggested that changes in diffusion may result from changes in extracellular and intracellular compartmental spaces. ${ }^{34}$ It is plausible that because cell growth is aberrant, there could be a further breakdown in the membrane structure that could contribute to a decrease in the overall number of barriers to water diffusion. In addition to the effects from myelin, anisotropic diffusion is linked to cellular structures such as neurofibrils and microtubules. Similarly, the axon membrane structure might play a significant role in diffusion anisotropy. ${ }^{3}$ A clear mechanism underlying allodynia needs to be investigated further; however, there is evidence from this study, and previous literature, that the stem cell injection produced histological changes. ${ }^{26}$

The changes in diffusion correspond to cellular structural changes produced from the stem cells and, therefore, we hypothesize that changes in diffusion can be used as a biomarker for the effects of stem cell transplantation. However, it is important to note that the structural changes detected using DTI are not specifically defined as functional repair. The allodynia denoted in this study may have occurred from the astrocytic population or C17.2 NSCs. ${ }^{22}$

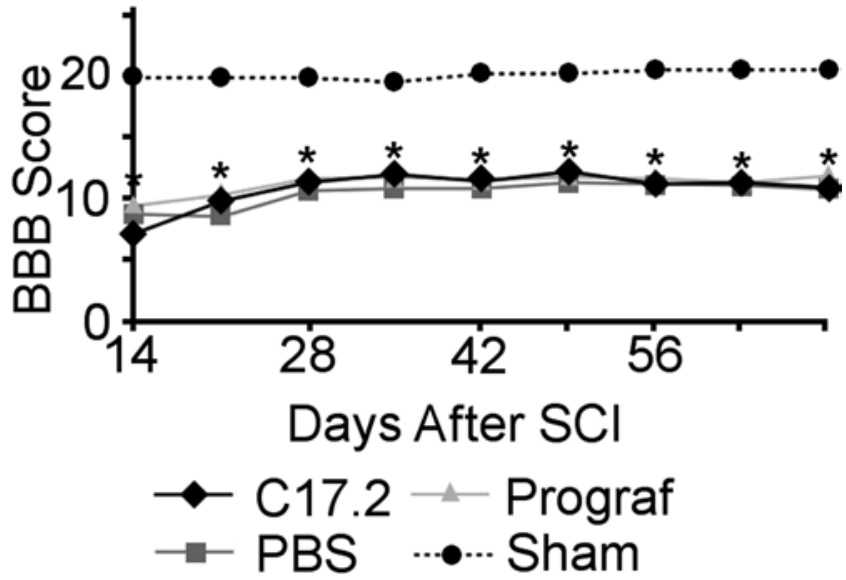

FIG. 5. BBB scoring from 2 weeks after a moderate SCI. Treatment groups started at 1 week postinjection and received no transplant solution, normal saline, normal saline with the immunosuppressant Prograf, or C17.2 NSC treatment with Prograf. The sham-injury group showed no motor deficits throughout the 10 -week course. Data are presented as combined hindlimb means. All treatment groups had a significant reduction in hindlimb motor capabilities compared with the sham group $\left({ }^{*} p<\right.$ $0.01)$, but not in comparison to each other $(p>0.05)$.

To the best of our knowledge, no study has examined the use of DTI to monitor the effect of cell transplantation at remote locations in the injured spinal cord. ${ }^{15}$ Instead, previous studies have used diffusion measurements to characterize the histological changes that occur after injury at the site of SCI. 7,932,37 Tagging superparamagnetic particles to neuronal cells can provide a means for tracking migration; ${ }^{5}$ however, in small regions of the body such as the spinal cord, cell labeling can result in susceptibility blooming. ${ }^{4}$ Other techniques in stem cell labeling involve the use of contrast agents, such as gadolinium that provides better spatial resolution, but the agents can carry risks to subjects with anaphylaxis or poor renal function. ${ }^{4,22-25} \mathrm{We}$ propose that our results in this study be used to build on previous work where spinal cord imaging remote from the

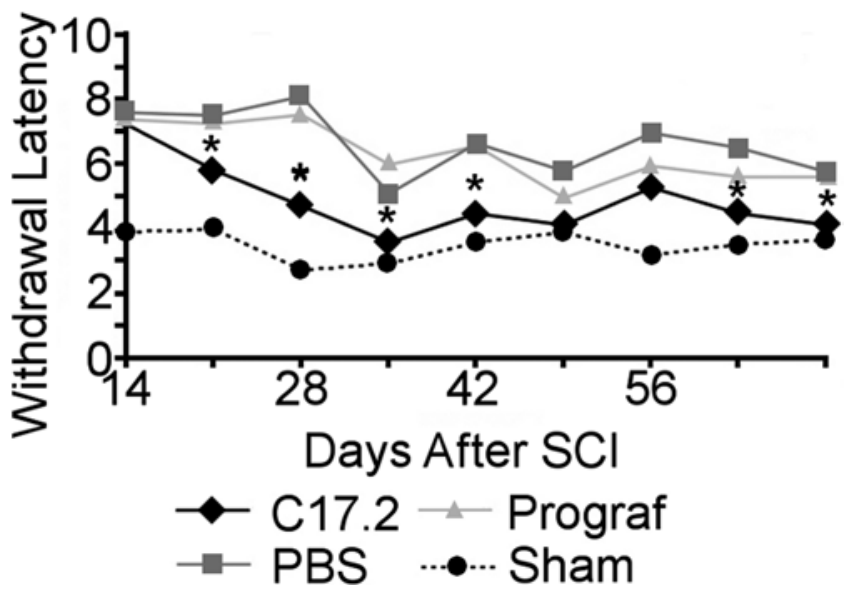

FIG. 6. Mean rat forelimb behavioral responses to a thermal stimulation of $55^{\circ} \mathrm{C}$. The $\mathrm{C} 17.2 \mathrm{NSC}$ transplant group showed a significantly reduced heat tolerance at $3-6,9$, and 10 weeks $(p<0.05)$ in comparison with the PBS and Prograf injection groups. Values on the $y$-axis represent seconds. 
site of injury is a tenable biomarker for the presence and severity of SCI. ${ }^{6,13}$

We have identified 3 potential limitations in our results. It is possible that the effect of the C17.2 NSCs could show diffusion changes that vary with the severity of the SCI. A logical extension of this work is to use the graded injury severity model that we have previously published to create a better spatiotemporal understanding of the microstructural changes that affect the diffusion of water. ${ }^{14}$ Another possible limitation to this study was the limited number of time points after the stem cell injection used to accurately portray the diffusion effects, especially those that occur long term. There is an initial inflammatory response after an SCI, but the continued axonal and Wallerian degeneration can extend for more than a year. ${ }^{14}$ Future studies would benefit from longer term time points in order to better understand how stem cells affect water diffusion throughout the spinal cord. Finally, tractography was not completed for this study. FA is a primary component of tractography modeling, and, due to the fact that there were no significant changes in FA measures, we felt that no additional information would be gained from tractography. In addition, Macias et al. noted that the allodynia that occurs after stem cell transplantation is associated with histological changes in the cervical segments of the spinal cord.22 Our study focused on just a few slices in the cervical section and thus tractography would have been of limited value, as data were collected over a small length of the spinal tract.

Limitations in imaging resolution early after injury could account for the in vivo results at Week 2 . It is possible that an increase in the number of directions or varying $b$ values would allow for a heightened resolution, which would be required to detect the acute changes that take place after the injection of transplant cells. Kim et al. showed that DTI is able to detect changes to the spinal cord that occur within 3 hours after an injury, suggesting that DTI has the potential to be sensitive to the immediate changes that occur after the injection of stem cells. ${ }^{17}$

Our results are the first to demonstrate that DTI detects changes in the underlying structure of the spinal cord after the transplantation of cells. DTI detected remote changes in the spinal cord structure related to previously published (and verified in this study) functional and immunohistochemical data. ${ }^{14}$ There was a significant increase in the overall diffusion of water in regions away from the injury site after 5 weeks postinjury that was associated with sensory measures of spinal cord function and histological evidence of cellular changes. This change in water diffusion detected by DTI persisted at 10 weeks after NSC transplants. While further refinement in image acquisition early after transplantation and the addition of later time points will assist in creating a better temporal profile of remote spinal cord structural changes after NSC transplants, we reason that this study represents an important advance in the development of a clinically available, noninvasive biomarker for future cell-based therapies for SCI.

\section{Conclusions}

We have demonstrated that DTI measures of diffusion in the cervical spinal cord increased with NSC transplantation, which also produced functional changes in the sensory systems of the forelimbs in rats. The increase in diffusion likely resulted from histological changes in the spinal cord due to cell proliferation and changes in cell morphology. These changes increased diffusivity, possibly due to decreases in the density of the membranous barriers to diffusion. We conclude that DTI measures of diffusion may be viable biomarkers of histological changes caused by transplanted cells, and future work that assesses the sensitivity of DTI to the reactivity of the implanted cells and severity of injury would be of great interest in the clinical setting.

\section{Acknowledgments}

This material is based upon work supported by the Falk Medical Trust Foundation, Advancing a Healthier Wisconsin endowment at the Medical College of Wisconsin, and the Department of Veterans Affairs.

\section{References}

1. Basser PJ, Pierpaoli C: Microstructural and physiological features of tissues elucidated by quantitative-diffusion-tensor MRI. J Magn Reson B 111:209-219, 1996

2. Basso DM, Beattie MS, Bresnahan JC: A sensitive and reliable locomotor rating scale for open field testing in rats. J Neurotrauma 12:1-21, 1995

3. Bavetta S, Hamlyn PJ, Burnstock G, Lieberman AR, Anderson PN: The effects of FK506 on dorsal column axons following spinal cord injury in adult rats: neuroprotection and local regeneration. Exp Neurol 158:382-393, 1999

4. Beaulieu C: The basis of anisotropic water diffusion in the nervous system - a technical review. NMR Biomed 15:435455, 2002

5. Brekke C, Williams SC, Price J, Thorsen F, Modo M: Cellular multiparametric MRI of neural stem cell therapy in a rat glioma model. Neuroimage 37:769-782, 2007

6. Bulte JW, Kraitchman DL: Iron oxide MR contrast agents for molecular and cellular imaging. NMR Biomed 17:484-499, 2004

7. Ellingson BM, Kurpad SN, Schmit BD: Ex vivo diffusion tensor imaging and quantitative tractography of the rat spinal cord during long-term recovery from moderate spinal contusion. J Magn Reson Imaging 28:1068-1079, 2008

8. Ellingson BM, Schmit BD, Kurpad SN: Lesion growth and degeneration patterns measured using diffusion tensor 9.4-T magnetic resonance imaging in rat spinal cord injury. J Neurosurg Spine 13:181-192, 2010

9. Ellingson BM, Ulmer JL, Kurpad SN, Schmit BD: Diffusion tensor MR imaging in chronic spinal cord injury. AJNR Am J Neuroradiol 29:1976-1982, 2008

10. Guleria S, Gupta RK, Saksena S, Chandra A, Srivastava RN, Husain M, et al: Retrograde Wallerian degeneration of cranial corticospinal tracts in cervical spinal cord injury patients using diffusion tensor imaging. J Neurosci Res 86:22712280, 2008

11. Huebner EA, Strittmatter SM: Axon regeneration in the peripheral and central nervous systems. Results Probl Cell Differ 48:339-351, 2009

12. Hofstetter CP, Holmström NA, Lilja JA, Schweinhardt P, Hao J, Spenger C, et al: Allodynia limits the usefulness of intraspinal neural stem cell grafts; directed differentiation improves outcome. Nat Neurosci 8:346-353, 2005

13. Jiang Q, Zhang ZG, Ding GL, Silver B, Zhang L, Meng H, et al: MRI detects white matter reorganization after neural progenitor cell treatment of stroke. Neuroimage 32:1080-1089, 2006

14. Jirjis MB, Kurpad SN, Schmit BD: Ex vivo diffusion tensor 
imaging of spinal cord injury in rats of varying degrees of severity. J Neurotrauma 30:1577-1586, 2013

15. Kakulas BA: Neuropathology: the foundation for new treatments in spinal cord injury. Spinal Cord 42:549-563, 2004

16. Keirstead HS, Nistor G, Bernal G, Totoiu M, Cloutier F, Sharp K, et al: Human embryonic stem cell-derived oligodendrocyte progenitor cell transplants remyelinate and restore locomotion after spinal cord injury. J Neurosci 25:4694-4705, 2005

17. Kim JH, Loy DN, Wang Q, Budde MD, Schmidt RE, Trinkaus K, et al: Diffusion tensor imaging at 3 hours after traumatic spinal cord injury predicts long-term locomotor recovery. J Neurotrauma 27:587-598, 2010

18. Kim JH, Song SK, Burke DA, Magnuson DS: Comprehensive locomotor outcomes correlate to hyperacute diffusion tensor measures after spinal cord injury in the adult rat. Exp Neurol 235:188-196, 2012

19. Lee-Kubli CA, Ingves M, Henry KW, Shiao R, Collyer E, Tuszynski MH, et al: Analysis of the behavioral, cellular and molecular characteristics of pain in severe rodent spinal cord injury. Exp Neurol 278:91-104, 2016

20. Loy DN, Kim JH, Xie M, Schmidt RE, Trinkaus K, Song SK: Diffusion tensor imaging predicts hyperacute spinal cord injury severity. J Neurotrauma 24:979-990, 2007

21. Lu P, Jones LL, Tuszynski MH: Axon regeneration through scars and into sites of chronic spinal cord injury. Exp Neurol 203:8-21, 2007

22. Macias MY, Syring MB, Pizzi MA, Crowe MJ, Alexanian AR, Kurpad SN: Pain with no gain: allodynia following neural stem cell transplantation in spinal cord injury. Exp Neurol 201:335-348, 2006

23. Magnitsky S, Watson DJ, Walton RM, Pickup S, Bulte JW, Wolfe JH, et al: In vivo and ex vivo MRI detection of localized and disseminated neural stem cell grafts in the mouse brain. Neuroimage 26:744-754, 2005

24. Modo M, Cash D, Mellodew K, Williams SC, Fraser SE, Meade TJ, et al: Tracking transplanted stem cell migration using bifunctional, contrast agent-enhanced, magnetic resonance imaging. Neuroimage 17:803-811, 2002

25. Modo M, Mellodew K, Cash D, Fraser SE, Meade TJ, Price $\mathrm{J}$, et al: Mapping transplanted stem cell migration after a stroke: a serial, in vivo magnetic resonance imaging study. Neuroimage 21:311-317, 2004

26. Moraes L, Vasconcelos-dos-Santos A, Santana FC, Godoy MA, Rosado-de-Castro PH, Jasmin, et al: Neuroprotective effects and magnetic resonance imaging of mesenchymal stem cells labeled with SPION in a rat model of Huntington's disease. Stem Cell Res (Amst) 9:143-155, 2012

27. Nevo U, Hauben E, Yoles E, Agranov E, Akselrod S, Schwartz M, et al: Diffusion anisotropy MRI for quantitative assessment of recovery in injured rat spinal cord. Magn Reson Med 45:1-9, 2001

28. Oku R, Satoh M, Fujii N, Otaka A, Yajima H, Takagi H: Calcitonin gene-related peptide promotes mechanical nociception by potentiating release of substance $\mathrm{P}$ from the spinal dorsal horn in rats. Brain Res 403:350-354, 1987

29. Ondarza AB, Ye Z, Hulsebosch CE: Direct evidence of primary afferent sprouting in distant segments following spinal cord injury in the rat: colocalization of GAP-43 and CGRP. Exp Neurol 184:373-380, 2003

30. Pierpaoli C, Barnett A, Pajevic S, Chen R, Penix LR, Virta A, et al: Water diffusion changes in Wallerian degeneration and their dependence on white matter architecture. Neuroimage 13:1174-1185, 2001
31. Ryder EF, Snyder EY, Cepko CL: Establishment and characterization of multipotent neural cell lines using retrovirus vector-mediated oncogene transfer. J Neurobiol 21:356-375, 1990

32. Schwartz ED, Chin CL, Shumsky JS, Jawad AF, Brown BK, Wehrli S, et al: Apparent diffusion coefficients in spinal cord transplants and surrounding white matter correlate with degree of axonal dieback after injury in rats. AJNR Am J Neuroradiol 26:7-18, 2005

33. Schwartz ED, Duda J, Shumsky JS, Cooper ET, Gee J: Spinal cord diffusion tensor imaging and fiber tracking can identify white matter tract disruption and glial scar orientation following lateral funiculotomy. J Neurotrauma 22:1388-1398, 2005

34. Schwartz ED, Hackney DB: Diffusion-weighted MRI and the evaluation of spinal cord axonal integrity following injury and treatment. Exp Neurol 184:570-589, 2003

35. Steindler DA: Neural stem cells, scaffolds, and chaperones. Nat Biotechnol 20:1091-1093, 2002

36. Snyder EY, Deitcher DL, Walsh C, Arnold-Aldea S, Hartwieg EA, Cepko CL: Multipotent neural cell lines can engraft and participate in development of mouse cerebellum. Cell 68:3351,1992

37. Sundberg LM, Herrera JJ, Narayana PA: In vivo longitudinal MRI and behavioral studies in experimental spinal cord injury. J Neurotrauma 27:1753-1767, 2010

38. Vande Velde G, Raman Rangarajan J, Vreys R, Guglielmetti C, Dresselaers T, Verhoye M, et al: Quantitative evaluation of MRI-based tracking of ferritin-labeled endogenous neural stem cell progeny in rodent brain. Neuroimage 62:367-380, 2012

39. Yin B, Tang Y, Ye J, Wu Y, Wang P, Huang L, et al: Sensitivity and specificity of in vivo diffusion-weighted MRI in acute spinal cord injury. J Clin Neurosci 17:1173-1179, 2010

\section{Disclosures}

The authors report no conflict of interest concerning the materials or methods used in this study or the findings specified in this paper.

\section{Author Contributions}

Conception and design: Kurpad, Jirjis, Schmit. Acquisition of data: Jirjis, Vedantam, Schmit. Analysis and interpretation of data: Kurpad, Jirjis, Valdez, Vedantam. Drafting the article: Kurpad, Jirjis, Valdez, Schmit. Critically revising the article: Kurpad, Jirjis, Valdez, Schmit. Reviewed submitted version of manuscript: Kurpad, Jirjis, Vedantam, Schmit. Approved the final version of the manuscript on behalf of all authors: Kurpad. Statistical analysis: Jirjis. Study supervision: Kurpad.

\section{Supplemental Information}

\section{Previous Presentations}

Portions of this work were presented in a poster form at Neuroscience 2012 for the Society for Neuroscience in New Orleans, Louisiana, October 2012.

\section{Correspondence}

Shekar N. Kurpad, Department of Neurosurgery, Medical College of Wisconsin, 8701 Watertown Plank Rd., Milwaukee, WI 53226. email: skurpad@mcw.edu. 